\title{
An eight-week yoga intervention is associated with improvements in pain, psychological functioning and mindfulness, and changes in cortisol levels in women with fibromyalgia
}

\author{
This article was published in the following Dove Press journal: \\ Journal of Pain Research \\ 25 July 2011 \\ Number of times this article has been viewed
}

\section{Kathryn Curtis \\ Anna Osadchuk Joel Katz}

Department of Psychology, Faculty of Health, York University, Toronto, ON, Canada
Correspondence: Katy Curtis Department of Psychology, BSB 297, York University 4700 Keele Street, Toronto, ON M3J IP3 Canada $\mathrm{Tel}+\mathrm{I} 6479955289$ $\mathrm{Fax}+\mathrm{I} 4167365814$ Email kjbc@yorku.ca or jkatz@yorku.ca
Objectives: Fibromyalgia (FM) is a chronic condition characterized by widespread musculoskeletal pain, fatigue, depression, and hypocortisolism. To date, published studies have not investigated the effects of yoga on cortisol in FM. This pilot study used a time series design to evaluate pain, psychological variables, mindfulness, and cortisol in women with FM before and after a yoga intervention.

Methods: Participants $(n=22)$ were recruited from the community to participate in a 75 minute yoga class twice weekly for 8 weeks. Questionnaires concerning pain (intensity, unpleasantness, quality, sum of local areas of pain, catastrophizing, acceptance, disability), anxiety, depression, and mindfulness were administered pre-, mid- and post-intervention. Salivary cortisol samples were collected three times a day for each of two days, pre- and post-intervention.

Results: Repeated measures analysis of variance (ANOVA) revealed that mean \pm standard deviation (SD) scores improved significantly $(p<0.05)$ from pre- to post-intervention for continuous pain (pre: $5.18 \pm 1.72$; post: $4.44 \pm 2.03$ ), pain catastrophizing (pre: $25.33 \pm 14.77$; post: $20.40 \pm 17.01$ ), pain acceptance (pre: $60.47 \pm 23.43$; post: $65.50 \pm 22.93$ ), and mindfulness (pre: $120.21 \pm 21.80$; post: $130.63 \pm 20.82$ ). Intention-to-treat analysis showed that median AUC for post-intervention cortisol (263.69) was significantly higher $(p<0.05)$ than median AUC for pre-intervention levels (189.46). Mediation analysis revealed that mid-intervention mindfulness scores significantly $(p<0.05)$ mediated the relationship between pre- and post-intervention pain catastrophizing scores.

Discussion: The results suggest that a yoga intervention may reduce pain and catastrophizing, increase acceptance and mindfulness, and alter total cortisol levels in women with FM. The changes in mindfulness and cortisol levels may provide preliminary evidence for mechanisms of a yoga program for women with FM. Future studies should use an RCT design with a larger sample size.

Keywords: fibromyalgia, pain, cortisol, yoga, psychological variables

\section{Introduction}

Fibromyalgia (FM) is a poorly understood condition that is characterized by widespread musculoskeletal pain and presents with other symptoms such as fatigue, cognitive dysfunction, sleep disturbances, anxiety, depression, gastrointestinal discomfort and stiffness. ${ }^{1}$ Recent proposed changes to the American College of Rheumatology (ACR) formal diagnostic criteria for FM include implementation of the widespread pain index (WPI) and symptom severity scale (SS), to supplement the previous gold standard of 
a tender point count, along with widespread pain for at least 3 months in at least 3 of the 4 quadrants of the body. ${ }^{2}$

Female gender, older age, low level of education, low socio-economic status, and divorce have been associated with FM. ${ }^{3}$ Psychological distress is a major risk factor for long term complaints ${ }^{4}$ and comorbidity with anxiety and depression is high, ${ }^{3}$ with rates of $13 \%-64 \%$ and $20 \%-80 \%$, respectively. ${ }^{5}$ Moreover, FM patients display generalized hypervigilance, ${ }^{6}$ characterized by a greater sensitivity to external stimuli in various modalities. Pain catastrophizing and low pain-self efficacy are other psychological factors that prevent healthy adjustment to $\mathrm{FM}^{7}$ and contribute to disability, ${ }^{8}$ pain, depression and quality of well being. ${ }^{9}$ Although the neurophysiological underpinnings of this disorder have yet to be elucidated, there is evidence that central sensitization is largely implicated in the extensive and enduring pain and that FM is accompanied by altered hypothalamic pituitary adrenal (HPA) axis and autonomic nervous system (ANS) functioning. ${ }^{3}$

Cortisol is a steroid hormone that is produced and released by the adrenal gland, and functions as a component of the hypothalamic-pituitary-adrenal (HPA) axis in response to stress or low blood sugar. Healthy HPA axis functioning, as measured by cortisol secretion, entails higher cortisol levels upon waking, a peak approximately 30-40 minutes postwaking (the cortisol awakening response [CAR]) and a decline over the course of the day, when levels reach a trough prior to sleep onset. ${ }^{10}$ Healthy functioning also involves HPA resilience, which refers to the ability of the HPA system to recover or rebound from stress. The literature on HPA functioning and cortisol levels in FM patients is equivocal. ${ }^{11}$ A host of variables complicates an accurate portrayal of HPA function, but variations in the methods used to collect cortisol samples, such as time of cortisol measurement (morning, afternoon, evening), number of measurements per day (1-8, or continuous readings), and source (salivary, plasma, serum or urinary) of cortisol, make it particularly difficult to derive a clear picture of HPA activity in FM patients. ${ }^{12}$

Notwithstanding the variability in cortisol sampling procedures and findings, it is accepted that there is a dysregulation of HPA functioning in patients with $\mathrm{FM},{ }^{11}$ resulting in alterations in levels of cortisol, corticotropin-releasing hormone, growth hormone and thyroid hormones, which may have secondary effects on pain, fatigue, immune function, mood and sleep. ${ }^{3}$ Recent research indicates that FM patients exhibit hypocortisolism, particularly as an attenuated CAR. ${ }^{13}$ Hypocortisolism is characterized by a blunted presentation of cortisol secretion, compromised HPA resilience and a triad of pain, fatigue and stress sensitivity. ${ }^{14}$ In support of this idea, women with FM have lower urinary ${ }^{15}$ and salivary cortisol levels ${ }^{16}$ than healthy controls, and less diurnal variability in comparison to individuals with rheumatoid arthritis. ${ }^{17}$ In addition, core features of FM, such as fatigue, pain, and psychological stress, have been associated with lower morning cortisol levels and blunted diurnal slopes. ${ }^{18-20}$ HPA axis dysfunction has also been associated with psychological dysfunction, such as depression, and stress but not with pain in patients with $\mathrm{FM},{ }^{12}$ indicating that the relationship between HPA function, pain and psychological distress in FM needs further examination.

Treatments for FM are general in nature and include the use of both pharmacological and non-pharmacological symptom management. Exercise is widely used as a treatment option and guidelines have been suggested for optimal results: minimize micromuscle trauma, minimize central sensitization, emphasize low-intensity exercise, individualize exercise, and maximize self-efficacy. ${ }^{21}$ Although research findings concerning isometric exercise and pain levels in FM are mixed, results from a randomized controlled trial indicate that cortisol levels in individuals with fibromyalgia do not differ from healthy controls when performing static muscular work and isometric contraction. ${ }^{22-24}$ Yoga has been used as a treatment for a variety of chronic conditions in which pain is a predominant feature. ${ }^{25}$ To date, there are only two published studies of yoga for the treatment of FM. ${ }^{26,27}$ Improvements were seen on measures of pain, fatigue, mood, pain catastrophizing, acceptance and coping in FM patients when compared to waitlist controls, and in pain scores when compared to a yoga plus touch condition. ${ }^{27}$ Findings from these studies suggest that yoga improves pain, fatigue and psychological variables in an FM population. Further investigation is needed to examine how yoga affects other psychological domains and functional capacity, such as mindfulness and pain disability. ${ }^{26}$

Mindfulness as a construct has garnered recognition for its therapeutic gains in a variety of pain related conditions. It is rooted in Buddhist and contemplative philosophies and is characterized by paying total attention to the present moment with a non-judgmental awareness of inner and outer experiences. ${ }^{28}$ Benefits of mindfulness practices for FM include the reduction of depressive symptoms mid- and posttreatment, ${ }^{29}$ decreases in sympathetic autonomic arousal after meditation, ${ }^{30}$ and reduced heat-induced pain intensity and unpleasantness ratings in response to slowed breathing practices. ${ }^{31}$ Since yoga encompasses elements of mindfulness and includes breathing techniques, it is possible that the benefits 
of yoga may be, in part, due to the evolving perspective and awareness that result from mindfulness.

Yoga may also exert its effects through physiological mechanisms, as it impacts the hypothalamic-pituitary-adrenal (HPA) axis. Specifically, morning levels of cortisol increased in both depressed patients who participated in a yoga intervention and in yoga practitioners when compared to controls, ${ }^{32,33}$ and cortisol levels decreased in undergraduate students after one yoga class, ${ }^{34}$ in yoga teachers over a oneday yoga intensive, ${ }^{35}$ in self-referred women after one yoga class, ${ }^{36}$ and in detoxifying youth after a yogic breathing program. ${ }^{37}$ Other studies did not report changes in cortisol post-yoga intervention. ${ }^{38-40}$ Taken together, the findings regarding the effects of yoga on HPA functioning are controversial and the use of standardized protocols for cortisol collection in research involving yoga interventions would be beneficial.

Although many studies of HPA functioning have been conducted in FM patients, the effects of a yoga intervention on cortisol levels in patients with FM have not yet been evaluated. Moreover, the effects of yoga on physiological, psychological, functional and pain variables have yet to be evaluated in a single study of patients with FM. Since yoga has been shown to alleviate many FM symptoms, such as pain and psychological functioning, and to improve HPA functioning in other populations, it is possible that yoga may improve HPA functioning, as measured by increased cortisol levels. The objectives of this pilot study were to evaluate the effects of an eight-week Hatha yoga intervention on measures of (1) pain (intensity, unpleasantness, quality, sum of local areas, pain catastrophizing, pain acceptance, pain disability), (2) general psychosocial functioning (anxiety, depression), (3) mindfulness, and (4) cortisol in a sample of patients with FM. It was hypothesized that the yoga program would result in improvements in pain and pain-related variables, psychological functioning, mindfulness, and increases of cortisol levels, greater diurnal variability, and a greater CAR.

\section{Materials and methods Subjects}

Due to the higher prevalence of FM in women than men, ${ }^{3}$ and the likelihood of disproportionate numbers of men and women in the yoga classes, only women were included in this study. The main inclusion criterion was written documentation of a diagnosis of FM by a physician. Exclusion criteria were: males with FM; a current yoga practice (or a recent practice in the past six months); self-reported or diagnosed bipolar, psychotic, or personality disorders; change in any medication in the month prior to the commencement of the saliva collection and yoga program; a habit of smoking more than 15 cigarettes a day; and currently pregnant or breastfeeding. Women with FM were recruited through public advertisements (postings in locations including government and school community centers, book stores, health food stores), support groups, health centers, and pain clinics in Toronto. Figure 1 shows the flow of participants through the study. Between April 2010 and May 2010, 57 women were screened, 24 of whom were eligible and available for the yoga classes and 19 completed the yoga program. The yoga program ran from June 16th to August 20th, 2010. The research protocol was reviewed and approved by the Human Participants Review Committee at York University prior to the start of the study.

\section{Procedure}

As depicted in Figure 2, the program consisted of several components: an information session; pre- and post-program saliva collection; yoga classes; a follow-up session; and administration of self-report questionnaires. Questionnaires were administered at three time (T) points: pre- (T1), mid(T2), and post- (T3) intervention.

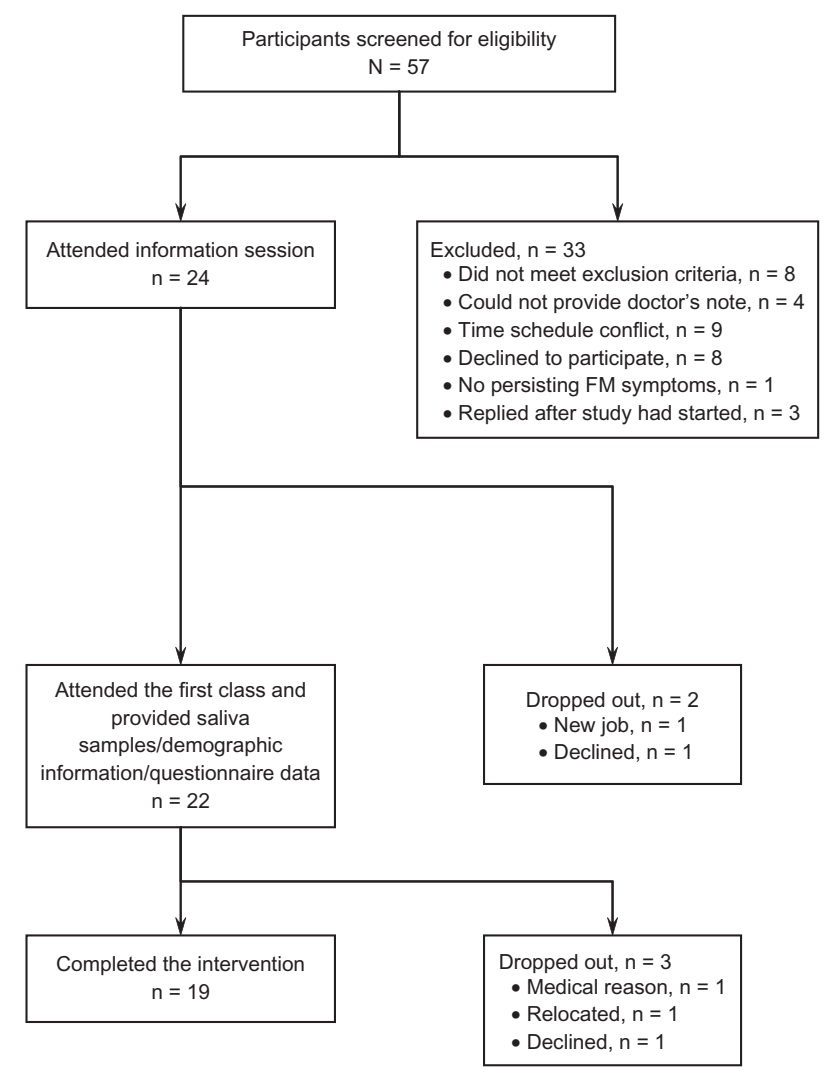

Figure I Participant flow through the course of the study. 


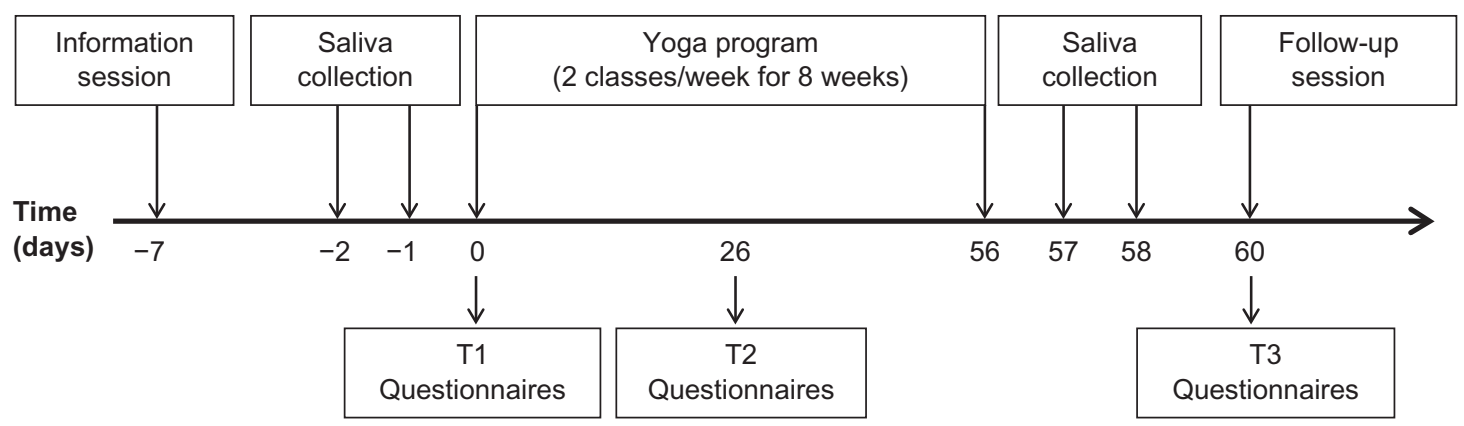

Figure 2 Order and time sequence of data collection. The information session was held seven days before the yoga program began. Saliva samples for cortisol analysis were collected on the two days before and the two days after the yoga program, for a total of four days. The yoga program ran for 8 weeks ( 2 classes/week) and the follow-up session took place two days after the final day of saliva collection. Questionnaires concerning pain and related variables, psychological factors and mindfulness were evaluated at three time points (TI, T2 and T3).

\section{Recruitment and information session}

Prospective participants who contacted the study coordinator were interviewed over the telephone using a script to determine eligibility. Those who met the eligibility criteria were emailed a general information letter about the program, the informed consent form, and a document outlining ways to decrease and prevent muscle soreness or tightness. The information letter outlined what was to be expected in terms of attendance, commitment, pacing, initial increases in pain due to overexertion, and how to handle flare-ups during the yoga program. Participants brought the consent form and a doctor's note confirming a diagnosis of FM to the information session, where they were provided with saliva collection kits and were given detailed instructions for taking samples. All participants were educated in the proper procedures for saliva collection, were given general instructions concerning the yoga program and were provided the opportunity to ask questions and discuss concerns.

\section{Saliva collection kits}

Saliva collection kits consisted of a booklet titled "Saliva Sample Collection and Information Instructions" with educational information about cortisol, labeled diagrams and explanations of the saliva collection equipment, step by step instructions for taking samples and recording information. ${ }^{41}$ Saliva sampling equipment (Salimetrics LLC, State College, PA) consisted of a small cotton swab, housed inside a centrifugation tube, in turn housed in a holding tube. All holding tubes were pre-labeled according to participant number, day $(1,2)$, and sample $(\mathrm{S} 1, \mathrm{~S} 2, \mathrm{~S} 3)$ number using freeze resistant labels provided by Salimetrics. Participants were provided with freezer bags labeled for each day of collection. The kits also contained a diary to record the date and time when each sample was taken, as well as questions about consumption of food, alcohol, caffeinated beverages, and drugs, and exercise activities around the time of sampling. Lastly, the kits contained a question and answer sheet relevant to commonly asked questions about saliva collection.

\section{Pre- and post-program salivary cortisol collection}

Participants took three pre-intervention samples on each of the two consecutive days prior to the start of the study, and another three samples on each of the two consecutive days beginning the day after the final yoga class, according to a two day sampling technique. ${ }^{42}$ The first sample (S1) was taken promptly upon awaking in the morning, the second sample (S2) was taken 30 minutes later, before brushing teeth, consuming food or beverages, or exercising, and the final sample (S3) was taken at night right before going to sleep. Participants were instructed not to exercise, consume drugs, alcohol, beverages or food within the hour before S3. All participants were called the night before the first day (pre- and post-intervention) of saliva sample collection to review the saliva sampling protocol and to answer any questions.

When taking a sample, participants were instructed to place a cotton swab under the tongue for at least 60 seconds, deposit the cotton swab into the centrifugation tube within the holding tube and store the entire sample in a freezer. Participants brought the pre- and post-intervention frozen samples to the studio on the first day of the yoga program and on the day of the scheduled follow-up session, respectively. The samples were transferred to a secure freezer until the end of the program, when both sets of samples were couriered to Salimetrics for cortisol immunoassay.

\section{Yoga classes}

Participants attended two 75-minute Hatha yoga classes each week for 8 weeks at Vidya Institute in Toronto. Classes were 
taught by a certified Yoga Alliance (Toronto, ON) instructor (KC) with 200 hours training. Hatha yoga is a mind-body practice that was developed in 15 th century India, in which physical yoga postures were practiced to prepare the mind for meditation. Theoretically, the term Hatha is composed of the Sanskrit terms "Ha" and "Tha", which refer to the sun, or heating and activating properties, and to the moon, or cooling or calming properties, respectively. By balancing these opposing qualities, physical and mental health are fostered and the development of self-awareness and mindbody unity are cultivated. ${ }^{43}$ Hatha yoga is an appropriate form of yoga for women with FM since it is gentle, can be easily modified to accommodate individual medical conditions and difficulties with mobility. The classes consisted of traditional, modified, and restorative yoga postures (asana), breathing exercises (pranayama), a brief meditation (dhyana), ${ }^{25}$ intention setting, mindfulness exercises and an introduction to the eight limbs of yoga. Participants were neither encouraged nor discouraged to practice asana at home between classes, and measures of home practice were not taken.

This program is similar in format to the Yoga as Awareness program developed by Carsen et $\mathrm{al}^{26}$ for women with FM, but differed in that short yoga philosophy teachings were given instead of dyadic presentations and that there was no group discussion in the present study. Teaching aspects of yoga philosophy, such as ahimsa (non-violence), aparigraha (nongrasping) and niyamas (guidance for one's relationships to the self) provided direction to participants for practicing the postures and breathing techniques, as recommended for chronic pain patients. ${ }^{44}$ Special care was taken in the formulation of instructions for practicing the postures so that they were tailored to individuals with FM, specific cues were given to provide participants with techniques for monitoring how to safely practice the postures when in pain and considerable instruction was given regarding the nature of a non-judgmental, compassionate, and accepting approach to practice.

All yoga classes began with philosophy teachings concerning the eight limbs of yoga, which are ethical considerations and guidelines to living a meaningful and purposeful life. These teachings were then practically applied to the physical yoga postures, as well as to a physical practice where pain is a predominant sensory experience. Modifications and variations in postures and purposeful sequencing were used to accommodate the pathophysiology and functional changes associated with FM. ${ }^{26}$ Slow transitions between asanas were used to address autonomic nervous system (ANS) dysregulation and restorative postures were interwoven throughout the class to calm the sympathetic nervous system. Low impact and intensity postures were used to minimize micromuscle trauma, and modified asanas (eg, sun salutation at the wall, variations of sitting postures) and props (blocks, straps and bolsters) were used to minimize musculoskeletal pain in the knees, back, hips, and wrists. Classes ended with corpse pose (savasana), which was accompanied by body scans and visualization practices, in order to help participants embrace relaxation, embody presence, and to serve as a focus for the mind, liberating it from attending to pain. ${ }^{44}$

\section{Follow-up session}

Three days after the final yoga class, participants attended a follow-up session at the studio, where they returned the frozen post-intervention saliva samples, completed the final set of questionnaires, and debriefed about their experiences. In specific cases where participants could not attend the follow-up session, individual meeting times were organized.

\section{Measures}

All participants were given a package of questionnaires at three time points to evaluate pain, mood, psychological functioning and mindfulness. The first set of questionnaires (T1) was completed at the yoga studio 45 minutes before the first class along with demographic and clinical information concerning occupation, education, ethnicity, medical or psychiatric illness, medication use and socioeconomic status. The second set (T2) was completed by participants at home or at the yoga studio before the ninth class, and the third set (T3) was filled out at the follow-up session. The study coordinator $(\mathrm{KC})$ was available to answer any questions and guide participants when completing the forms. Each questionnaire package consisted of the following measures.

\section{McGill Pain Questionnaire short-form 2 (MPQ-SF-2)}

The MPQ-SF-2 ${ }^{45}$ is a multi-dimensional measure of pain that consists of 22 pain descriptors. Participants rate each descriptor on an 11-point scale ranging from 0 ("none") to 10 ("worst possible"). Subscale scores are computed by calculating mean ratings for the following subscales: (1) continuous pain, (2) intermittent pain, (3) neuropathic pain, and (4) affective descriptors. Total score is the mean of the four subscale scores. The MPQ-SF-2 has very good to excellent psychometric properties, including adequate to high internal consistency reliability estimates for the four subscales $(0.73-0.87)$ and the total score (0.91-0.95), good construct validity, and sensitivity to change in response to pharmacotherapy. ${ }^{45}$ 


\section{Numeric Rating Scale (NRS) for pain} intensity $(\mathrm{I})$ and unpleasantness $(\mathrm{U})$

The $\mathrm{NRS}^{46}$ provided a global measure of pain intensity and pain unpleasantness. Participants were instructed to rate the general level of pain intensity and pain unpleasantness experienced over the past week on an 11-point scale with endpoints of 0 ("no pain") and 10 ("the most intense pain sensation imaginable") for NRS-I, and 0 ("not unpleasant") and 10 ("the most unpleasant sensation imaginable") for NRS-U. NRSs for pain have good construct validity, ${ }^{46}$ show significant, positive correlations with other measures of pain, and are sensitive to change in response to treatment. $^{46}$

\section{Sum of Local Areas of Pain (SLAP)}

The $\mathrm{SLAP}^{47}$ consists of diagrams of the human body depicting both the front and the back view divided into 20 areas, 18 of which include standard FM tender points with an additional two areas on the abdomen. The SLAP also consists of many of the same areas as the WPI, ${ }^{2}$ which measures 19 body regions for pain, although the two measures divide body regions differently. For instance, the SLAP measures the front and back of each arm as separate regions, while the WPI measures the upper and lower portions of each arm. Participants are instructed to circle areas in which they have been experiencing pain over the past week. The SLAP was selected for this study since (1) the practicality, accuracy and reliability of using tender points to determine pain intensity in FM patients has been questioned, ${ }^{2}(2)$ tender point count requires specialized training, and (3) the SLAP has been shown to be a better clinical predictor of pain than tender point count in FM patients. ${ }^{47}$

\section{Pain Catastrophizing Scale (PCS)}

The $\mathrm{PCS}^{48}$ is a 13 -item self-report questionnaire that measures catastrophic thinking in relation to experienced or anticipated pain. Participants are asked to read each item and indicate the extent to which they experience certain thoughts and feelings when experiencing pain by selecting a number from 0 ("not at all") to 4 ("all the time"). Scores range from 0-52, with higher scores reflecting higher levels of pain catastrophizing. The PCS yields a total score and three subscale scores assessing rumination (focus on pain sensations), magnification (exaggerating the threat value of pain sensations) and helplessness (perceiving oneself as unable to cope with pain symptoms). The PCS has high internal consistency (coefficient alphas: total PCS $=0.87$, rumination $=0.87$, magnification $=0.66$, and helplessness $=0.78$ ).

\section{Pain Disability Index (PDI)}

The $\mathrm{PDI}^{49}$ is a self-report questionnaire that measures the extent to which pain interferes with seven daily activities and life domains. Each domain is rated on an 11-point scale ranging from 0 ("no disability") to 10 ("total disability"). The total score ranges from 0 to 70 , with higher scores indicating more pain disability. The PDI has high internal consistency ( $\alpha=0.86)$, modest test-re-test reliability ( $\alpha=0.44)$, and good concurrent validity based on significant correlations with objective measures of pain related disability. ${ }^{49}$

\section{Hospital Anxiety and Depression Scale (HADS)}

The $\operatorname{HADS}^{50}$ is a 14-item self-report questionnaire that measures symptoms of anxiety ( 7 items) and depression ( 7 items). For each item, participants are asked to select one from among four possible choices (scored from 0 to 3 ) that best describes how they have been feeling over the past week. The HADS yields an anxiety (HADS-A) and a depression (HADS-D) subscale score, each with a maximum total score of 21. Internal consistency is high for both the HADS-A $(\alpha=0.83)$ and HADS-D $(\alpha=0.82)$ subscales. ${ }^{51}$ Concurrent validity of the HADS is very good, as measured by correlation coefficients of between 0.62 and 0.73 for the HADS-D with various well-validated depression scales and correlation coefficients of between 0.49 and 0.81 for the HADS-A with various well-validated anxiety measures. ${ }^{51}$

\section{Five Facet Mindfulness Questionnaire (FFMQ)}

The FFMQ ${ }^{52}$ is a 39-item self-report questionnaire that measures levels of mindfulness according to five facets: observing, describing, acting with awareness, non-judging of inner experience, and non-reactivity to inner experience. Participants respond to each item by selecting the number that is "most generally true" of his/her experience, on a scale of 1 ("never or rarely true") to 5 ("very often or always true"). Total scores range from 0 to 195 . Higher scores indicate greater levels of mindfulness. Each subscale of the FFMQ describes different areas of mindfulness and is correlated with different aspects of psychological functioning. For instance, the describing facet is most positively correlated with emotional intelligence and negatively correlated with alexithymia, which is characterized by difficulty identifying physical symptoms as somatic representations of emotions, while the non-reactivity facet is most positively correlated with self-compassion, and the observing facet with openness. ${ }^{52}$ The FFMQ is based on a factor analytic study of five independently developed mindfulness questionnaires, with good internal consistency ${ }^{52}$ and construct validity. ${ }^{53}$ 


\section{Chronic Pain Acceptance Questionnaire (CPAQ)}

The $\mathrm{CPAQ}^{54}$ is a 20 -item questionnaire that measures how participants react and adapt to living with chronic pain. Participants are asked to respond to "how true" each item is by selecting a number from 0 ("never true") to 6 ("always true"). The items load on a two-factor model including activity engagement and pain willingness (recognition that pain avoidance is not a helpful way to cope). ${ }^{54}$ The CPAQ has adequate internal consistency (Cronbach's $\alpha$ : total $=0.78$, activity engagement $=0.78$, pain willingness $=0.85$ ). Both factors significantly predict pain-related disability and distress. ${ }^{54}$

\section{Salivary cortisol measurement}

All saliva samples were assayed for cortisol in duplicate using a highly sensitive enzyme immunoassay (Salimetrics, State College, PA). The test used $25 \mu \mathrm{L}$ of saliva per determination, has a lower limit of sensitivity of $0.003 \mu \mathrm{g} / \mathrm{dL}$, standard curve range from $0.012 \mu \mathrm{g} / \mathrm{dL}$ to $3.0 \mu \mathrm{g} / \mathrm{dL}$, an average intra-assay coefficient of variation of $3.5 \%$ and an average inter-assay coefficient of variation of $5.1 \%$. Method accuracy, determined by spike and recovery, averaged $100.8 \%$, and linearity, determined by serial dilution, averaged $91.7 \%$. Values from matched serum and saliva samples show the expected strong linear relationship, $r(47)=0.91, p<0.0001$. A random selection of $10 \%$ of the samples was analyzed twice in order to determine reliability of the assay.

\section{Data preparation and analysis}

After examining the salivary cortisol levels $(\mu \mathrm{g} / \mathrm{dL})$ for normality and outliers (described below) the data were averaged across the two sampling days to produce one value for each participant at each of the three sampling points, for both preand post-intervention, yielding six values for each participant. Based on previous research, three different cortisol measures were calculated: (1) total area under the curve (AUC), ${ }^{55}$ which provides information about total cortisol output, (2) CAR, calculated as the difference between $\mathrm{S} 2$ and $\mathrm{S} 1,{ }^{19}$ and (3) diurnal change, calculated as the difference between S1 and S3. ${ }^{56}$ The AUC was calculated separately for each of the four collection days, then the two pre-intervention AUCs were averaged to produce one pre-intervention AUC value for each participant and similarly, the two post-intervention AUCs were averaged to produce one post-intervention AUC value for each participant.

Statistical analysis was performed with SPSS (Somers, NY) version 19.0. Demographic, clinical and diary-related variables were analyzed using the descriptive and the explore functions. The raw data was explored for skew and was analyzed using the Shapiro-Wilk test for normality and Levene's test for homogeneity of variance. Pre- and postvalues of the AUC, the CAR and diurnal change were compared using paired samples $t$-tests or Wilcoxon signed-rank tests, depending on violations of assumptions associated with the former test.

All self-report measures were assessed for normality using the Shapiro-Wilk test. Self-report data were analyzed using grouped repeated measures MANOVAs, which were followed by one-way repeated measures ANOVAs (T1, T2, T3) and LSD post hoc analysis in the presence of a significant main effect of time. ${ }^{57}$ Sphericity was evaluated using Bartlett's test and in all cases of violation, Huynh-Feldt adjustments were used.

Simple mediation analysis was conducted using a bootstrapping approach (2,000 resamples), as recommended for small sample sizes ${ }^{58}$ to evaluate the mediating effect of total FFMQ scores at T2 on the relationship between PCS scores at $\mathrm{T} 1$ and $\mathrm{T} 3$.

Data are reported as mean \pm SD unless otherwise stated.

\section{Results}

\section{Demographic and clinical variables}

The characteristics of the sample are summarized in Table 1. The mean age was $47.4 \pm 13.7$ years (range: $17-71$ years); height was $163.6 \pm 8.43 \mathrm{~cm}$ (range: $142.2-177.8 \mathrm{~cm}$ ) and weight was $70.98 \mathrm{~kg} \pm 18.89$ (range: $53.5-132.9 \mathrm{~kg}$ ). Participants had been diagnosed with FM for an average of $13.2 \pm 8.6$ years (range: $1-27$ years). Participant use of various pain treatments (pharmacological, natural health products, physical approaches, psychological approaches and medical interventions) are displayed in Table 2. The mean \pm SD number of yoga classes attended was $12.9 \pm 2.02$ (out of 15 ) and $86.36 \%$ of the participants completed the yoga program.

\section{Treatment results}

\section{Repeated measures analysis for pain and related} variables, psychological factors and mindfulness

Three repeated measures MANOVAs were conducted for groups of similar variables: (1) pain variables (NRS-I, NRS-U, MPQ-SF-2, SLAP), (2) pain-related variables (PCS, CPAQ, PDI) and (3) mood and mindfulness variables (HADS-D, HADS-A, FFMQ), to determine changes in those sets of dependent variables over time. A significant multivariate time effect was found for pain-related and mood and mindfulness variables, $V=0.42, F(6.0,66.0)=2.92, p<0.05$, and $V=0.36$, 
Table I Demographic and clinical variables at baseline $(\mathrm{N}=22)$

\begin{tabular}{|c|c|}
\hline Demographic & $\mathbf{N}(\%) / M(S D)$ \\
\hline Age & $47.4(13.73)$ \\
\hline Height & $163.58(8.43)$ \\
\hline Weight (kg) & $70.98(18.89)$ \\
\hline Body mass index (BMI) & $26.48(6.27)$ \\
\hline Years since diagnosis & $13.16(8.55)$ \\
\hline \multicolumn{2}{|l|}{ Race/ethnicity } \\
\hline African Canadian & I (4.5\%) \\
\hline South Asian & I (4.5\%) \\
\hline East Asian & I (4.5\%) \\
\hline Middle Eastern/north & I (4.5\%) \\
\hline Caucasian & $16(72.7 \%)$ \\
\hline Hispanic & I (4.5\%) \\
\hline West Indian & I (4.5\%) \\
\hline \multicolumn{2}{|l|}{ Socioeconomic class } \\
\hline High & $0(0.0 \%)$ \\
\hline Middle-high & I (4.5\%) \\
\hline Middle & $13(59.1 \%)$ \\
\hline Middle-low & $2(9.1 \%)$ \\
\hline Low & $5(22.7 \%)$ \\
\hline Employed & $8(36.4 \%)$ \\
\hline \multicolumn{2}{|l|}{ Level of education } \\
\hline Grade-school & I (4.5\%) \\
\hline High-school & $3(13.6 \%)$ \\
\hline University/college & $14(63.6 \%)$ \\
\hline Post-graduate school & $4(18.2 \%)$ \\
\hline Smokes cigarettes & $4(18.2 \%)$ \\
\hline Major life event in past year & II (50.0\%) \\
\hline Presence of other ongoing & $16(72.6 \%)$ \\
\hline \multicolumn{2}{|l|}{ pain problems } \\
\hline \multicolumn{2}{|l|}{ Fibromyalgia pain frequency } \\
\hline Daily & $15(68.2 \%)$ \\
\hline Weekly & $4(18.2 \%)$ \\
\hline Monthly & 0 \\
\hline Other & $3(13.6 \%)$ \\
\hline \multicolumn{2}{|l|}{ Average pain intensity } \\
\hline Mild & $4(18.2 \%)$ \\
\hline Moderate & $9(40.9 \%)$ \\
\hline Severe & 9 (40.9\%) \\
\hline
\end{tabular}

$F(6.0,66.0)=2.44, p<0.05$, respectively, but not for the pain variables, $V=0.17, F(8.0,64.0)=0.75, p>0.05$. Univariate ANOVAs were conducted for all measures, including the pain variables, as it was possible that the small sample size may have contributed to the lack of significance in this grouping.

Table 3 shows the means and SDs for each measure across the three time points, as well as significant $p$ values. HuynhFeldt adjusted F-tests revealed a significant main effect of time for PCS-total, $F(2.0,34.0)=3.46, p<0.05, \eta_{\mathrm{p}}{ }^{2}=0.17$, PCS-helplessness, $F(1.9,31.8)=3.77, p<0.05, \eta_{\mathrm{p}}^{2}=0.18$, CPAQ-total, $F(1.8,30.7)=7.53, p<0.05, \eta_{\mathrm{p}}{ }^{2}=0.31$, CPAQpain willingness, $F(2.0,34.0)=3.46, p<0.05, \eta_{\mathrm{p}}{ }^{2}=0.17$, CPAQ-activity engagement, $F(2.0,34.0)=9.04, p<0.05$,
Table 2 Pain medications and treatments previously or currently used $(\mathrm{N}=22)$

\begin{tabular}{|c|c|c|}
\hline Pain medication and treatments & $\mathbf{N}$ & $(\%)$ \\
\hline Pharmacological medications & 18 & $(81.8)$ \\
\hline Opioid based medications & 9 & $(40.9)$ \\
\hline Non-steroidal anti-inflammatory drugs & 10 & $(45.5)$ \\
\hline Anti-convulsant drugs & 4 & $(18.2)$ \\
\hline Acetaminophen & 8 & $(36.4)$ \\
\hline Antidepressants & 3 & $(13.6)$ \\
\hline Natural health products & 18 & $(81.8)$ \\
\hline \multicolumn{3}{|l|}{$\begin{array}{l}\text { Supplements and vitamins, marijuana, } \\
\text { herbs, homeopathy, essential oils, etc }\end{array}$} \\
\hline Physical approaches & 20 & $(90.9)$ \\
\hline \multicolumn{3}{|l|}{$\begin{array}{l}\text { Massage, acupuncture, Tai Chi, } \\
\text { physiotherapy, chiropracty, } \\
\text { craniosacral treatment, etc }\end{array}$} \\
\hline Psychological approaches & 17 & $(77.3)$ \\
\hline \multicolumn{3}{|l|}{$\begin{array}{l}\text { Meditation, breathing exercises, } \\
\text { psychotherapy, distraction, relaxation, } \\
\text { religion, hypnosis, etc }\end{array}$} \\
\hline Medical interventions & 6 & $(27.3)$ \\
\hline Cortisone, anesthetic injections & & \\
\hline
\end{tabular}

$\eta_{\mathrm{p}}{ }^{2}=0.35$, MPQ-SF2-continous pain, $F(2.0,36.0)=3.84$, $p<0.05, \eta_{\mathrm{p}}^{2}=0.18$, FFMQ-total, $F(1.4,25.7)=5.45, p<0.05$, $\eta_{\mathrm{p}}{ }^{2}=0.23$, FFMQ-describing, $F(1.7,30.5)=4.02, p<0.05$, $\eta_{\mathrm{p}}{ }^{2}=0.18$, and FFMQ-non-reactivity, $F(1.4,24.3)=6.15$, $p<0.05, \eta_{\mathrm{p}}{ }^{2}=0.26$. LSD comparisons revealed significant improvement $(p<0.05)$ between T1 and T2 for FFMQ-total, FFMQ-non-reactivity, CPAQ-total, CPAQ-activity engagement, and MPQ-SF-2-continuous pain, between T1 and T3 for PCS-total, PCS-helplessness, FFMQ-total, FFMQdescribing, FFMQ-non-reactivity, CPAQ-total, CPAQ-activity engagement, MPQ-SF-2-continuous pain, and between T2 and T3 FFMQ-describing, CPAQ-total, and CPAQ-pain willingness.

Mediation analysis revealed that scores on the FFMQ at T2 partially, but significantly, mediated the relationship between scores on the PCS at T1 and T3. Mid-intervention mindfulness scores were significantly related to end-oftreatment pain catastrophizing scores $(F(1.0,17.0)=33.9$, $p=0.000 ; r=0.82)$ and the relationship between PCS at T1 and T3 $(F(1.0,17.0)=35.1, p=0.000 ; r=0.82)$ was reduced (by $r^{2}=0.35$ ) after controlling for FFMQ at T2 (F change $(1.0,16.0)=7.4, p=0.02$; partial $r=0.56)$. Furthermore, both the Sobel test (Sobel value $=0.40, \mathrm{SEM}=0.18 ; z=2.22$, $\left.p=0.03, \mathrm{CI}_{95}=0.05,0.75\right)$ and the bootstrapping analysis $\left(\right.$ Mean $\left.=0.38, \mathrm{SEM}=0.17 ; \mathrm{CI}_{.95}=0.02,0.74\right)$ showed that the total effect of PCS scores at baseline on PCS scores at the end of the intervention was significantly reduced once 
Table 3 Mean (SD) values for pain, psychological and mindfulness variables across time $(\mathrm{N}=19)$

\begin{tabular}{|c|c|c|c|c|}
\hline Measure & $\begin{array}{l}\text { Pre-intervention } \\
\text { (TI) }\end{array}$ & $\begin{array}{l}\text { Mid-intervention } \\
\text { (T2) }\end{array}$ & $\begin{array}{l}\text { Post-intervention } \\
\text { (T3) }\end{array}$ & $\begin{array}{l}\text { Significance } \\
\text { ( } p \text { value) }\end{array}$ \\
\hline NRS-I & $5.21(2.80)$ & $4.68(3.00)$ & $4.68(2.75)$ & ns \\
\hline NRS-U & $5.33(2.89)$ & $4.50(3.37)$ & $4.67(2.63)$ & ns \\
\hline SLAP & II.89 (5.50) & $12.39(5.27)$ & $12.83(5.140)$ & ns \\
\hline FFMQ-totala & $|20.2|(2 \mid .80)$ & I26.47 (22.53) & I $30.63(20.82)$ & 0.02 \\
\hline FFMQ-observing & $29.32(4.77)$ & $29.61(4.75)$ & $30.89(4.22)$ & ns \\
\hline FFMQ-describing ${ }^{\mathrm{b}, \mathrm{c}}$ & $23.58(7.02)$ & $23.87(7.87)$ & $25.84(7.3 I)$ & 0.03 \\
\hline FFMQ-acting with awareness & $18.13(5.04)$ & $19.26(4.91)$ & $20.08(4.80)$ & ns \\
\hline FFMQ-non-judging & $23.84(5.52)$ & $25.55(5.01)$ & $25.37(5.5 \mathrm{I})$ & ns \\
\hline FFMQ-non-reactivitya,b & $19.16(5.48)$ & $21.53(4.59)$ & $22.00(4.6 \mathrm{I})$ & 0.01 \\
\hline PDI & $38.14(17.17)$ & $36.83(16.31)$ & $33.81(14.43)$ & ns \\
\hline PCS-total ${ }^{b}$ & $25.33(14.77)$ & $21.67(16.26)$ & $20.40(17.01)$ & 0.04 \\
\hline PCS-helplessness ${ }^{b}$ & $11.50(7.43)$ & $9.72(7.47)$ & $9.28(7.83)$ & 0.04 \\
\hline PCS-magnification & $5.94(3.45)$ & $4.8 I(3.5 I)$ & $4.69(3.18)$ & ns \\
\hline PCS-rumination & $8.67(4.83)$ & $7.67(5.72)$ & $7.44(5.89)$ & ns \\
\hline CPAQ-totala ${ }^{\mathrm{a}, \mathrm{b}, \mathrm{c}}$ & $60.47(23.43)$ & $65.44(19.20)$ & $69.50(22.93)$ & 0.003 \\
\hline CPAQ-activity engagement $\mathrm{t}^{\mathrm{a}, \mathrm{b}}$ & $37.86(15.13)$ & $43.83(11.58)$ & 44.75 (I4.57) & 0.001 \\
\hline CPAQ-pain willingness ${ }^{c}$ & $22.6 \mathrm{I}(10.35)$ & $21.61(9.53)$ & $24.75(10.72)$ & 0.04 \\
\hline MPQ-SF-2-total & $4.03(1.86)$ & $3.38(1.73)$ & $3.62(2.14)$ & ns \\
\hline MPQ-SF-2-continuous pain ${ }^{a, b}$ & $5.18(1.72)$ & $4.44(2.03)$ & $4.43(2.14)$ & 0.03 \\
\hline MPQ-SF-2-intermittent pain & $3.32(2.18)$ & $2.57(2.02)$ & $3.40(2.45)$ & ns \\
\hline MPQ-SF-2-neuropathic pain & $3.5 I(2.10)$ & $3.20(1.85)$ & $3.01(2.25)$ & ns \\
\hline MPQ-SF-2-affective pain & $4.26(2.45)$ & $3.38(2.49)$ & $3.64(2.72)$ & ns \\
\hline HADS-A & $10.83(4.40)$ & $11.50(5.06)$ & $9.78(4.47)$ & ns \\
\hline HADS-D & $9.00(4.54)$ & $8.65(4.65)$ & $7.47(4.26)$ & ns \\
\hline
\end{tabular}

Note: Huynh-Feldt adjusted F-tests for significant main effects of time were conducted for all self-reported measures. Numeric rating scale for pain intensity: NRS-I; numeric rating scale for unpleasantness: NRS-U; Sum of Local Areas of Pain: SLAP; Five Facet Mindfulness Questionnaire: FFMQ; Pain Disability Index: PDI, Pain Catastrophizing Scale: PCS, Chronic Pain Acceptance Questionnaire: CPAQ; McGill Pain Questionnaire short form 2: MPQ-SF-20; Hospital Anxiety and Depression-Depression Scale: HADS-D, Hospital Anxiety and Depression Scale-Anxiety: HADS-A. ${ }^{a} p<0.05$ for TI vs T2; ${ }^{b} p<0.05$ for TI vs T3; and ${ }^{c} p<0.05$ for T2 vs T3.

the mediator (ie, FFMQ scores at mid-intervention) was added to the model.

\section{Cortisol analysis}

As expected, reliability analysis performed on a randomly selected $10 \%$ of the cortisol samples showed very high between-sample correlations and did not show a significant difference between samples $(t(22)=1.98, p<0.05, r=0.98)$. Three cortisol samples were missing and were replaced with the value associated with the equivalent time point on the adjacent day of sampling. One participant underwent a medical procedure requiring her to fast before one of the days of sampling, and so data collected for that day were not used. Figure 3 shows pre- and post-yoga intervention mean salivary cortisol levels for S1, S2, and S3 averaged across the two sampling days.

The distributions of six of the 12 sampling time points (M1, M2, and M3 for the two days pre- and post-intervention) were skewed and five of the 12 sampling time points were non-normal according to the Shapiro-Wilk test $(p<0.05)$. Consequently the cortisol data were analyzed by nonparametric Wilcoxon signed-rank tests.
Using a protocol-compliant analysis based on data from the 19 participants who completed the study, post-intervention cortisol AUC (median $=230.20$ ) was greater than preintervention cortisol AUC (median $=189.46), z=-1.77$,

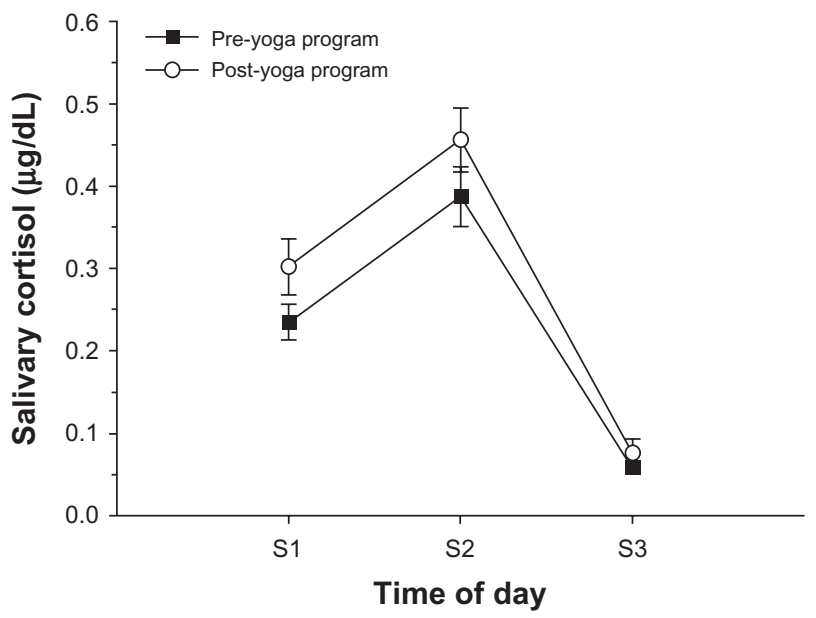

Figure 3 Pre- and post-intervention means for salivary cortisol levels $(\mu \mathrm{g} / \mathrm{dL})$, averaged across the two sampling days. SI, S2, S3 indicate first sample upon wakening, second sample 30 minutes after awakening, and third sample before sleep, respectively. Two outliers from the raw data that had z-scores of greater than \pm 3 were adjusted to values that were \pm 2 standard deviations from the mean of that measurement. 
$p=0.08$ but this did not reach the conventional $p<0.05$ level of significance. In contrast, using an intention-to-treat analysis based on the total sample $(n=22)$, in which the missing postintervention data for the three participants who dropped out of the study were estimated using the series mean, post-intervention AUC (median =263.69) was significantly greater than pre-intervention AUC (median $=189.46), z=-2.45$, $p<0.05$.

Median pre-intervention CAR $(0.15 \mu \mathrm{g} / \mathrm{dL})$ and diurnal change $(0.18 \mu \mathrm{g} / \mathrm{dL})$ did not differ significantly from the median post-intervention CAR $(0.14 \mu \mathrm{g} / \mathrm{dL})$ or diurnal change $(0.18 \mu \mathrm{g} / \mathrm{dL}), z=-0.20$ and $z=-0.77, p>0.05$, respectively.

\section{Self-report diaries and adherence}

Self-reported adherence to saliva sampling was $99.6 \%$. In accordance with instructions, no participants reported engaging in physical exercise or consumed any alcohol, caffeinated beverages, or food in the hour prior to taking S3 on each of the four days of collection, as recorded in the cortisol diaries.

\section{Discussion}

This pilot study evaluated the effects of an eight-week yoga intervention on a variety of symptoms in a sample of women with FM. The results demonstrated post-intervention improvement on a variety of pain and psychological variables, including continuous pain, pain catastrophizing, pain helplessness, chronic pain acceptance, activity engagement, pain willingness, mindfulness, non-reactivity to inner experience and ability to describe inner experience. Moreover, using an intention to treat analysis, total cortisol output increased significantly post-intervention. These results suggest that a Hatha yoga program specifically tailored to the needs of a female FM population reduces continuous pain, pain catastrophizing and increases chronic pain acceptance and mindfulness.

Improvements in pain, pain catastrophizing and chronic pain acceptance are consistent with the results of a recently published Yoga as Awareness intervention for women with FM, which showed a reduction in pain, fatigue, pain catastrophizing, and an increase in acceptance, mood and coping. ${ }^{26}$ The present results confirm many of these recent findings and extend them by suggesting that the pre- to postintervention changes we observed in mindfulness and cortisol levels may point to potential mechanisms of the present yoga program for women with FM.

The therapeutic benefits of mindfulness have been attributed to three components: intention, attention and attitude. ${ }^{59}$
These components serve to generate awareness and purpose for certain behaviors, to facilitate observation of both internal and external experiences while withholding judgment about the nature of those experiences, and to enhance the quality of how one attends. Kindness, openness and acceptance are pivotal to cultivating a state of non-striving, in which one can let go of hoping for pleasant experiences. Shapiro et al posit that these three elements contribute to a large proportion of the variance in the transformations that result from mindfulness practice in terms of psychological functioning, pain, and other outcomes. ${ }^{59}$ These considerations are supported by the results of the mediation analysis showing that mid-intervention levels of mindfulness (which increased significantly from baseline to T2 as shown in Table 3) mediated the reduction in pain catastrophizing from pre- to post- intervention. The results of the mediation analysis support recent fear-avoidance models of chronic pain, which propose that chronic pain is in part maintained by fear of pain and pain catastrophizing through the negatively reinforcing effects of avoiding (eg, behavioral, emotional, interoceptive) pain-related cues and sensations. ${ }^{60}$ We suggest that the in-class emphasis that mindfulness practices placed on attending, without judgment, to pain and the ensuing cognitive-emotional reactions (eg, catastrophic thoughts), permitted participants to circumvent their typical avoidance strategies and to experience the pain and distress, thereby reducing catastrophic appraisals by a process of interoceptive exposure. Furthermore, it is possible that the mindfulness practices and their effects on reducing pain catastrophizing may have contributed to the significant increases in chronic pain acceptance observed over the course of the intervention, but further research is needed to better understand these relationships.

Given that HPA axis dysregulation in FM has received considerable attention, the present results showing increased levels of total cortisol output (AUC) is especially noteworthy. This change suggests that the yoga program may have contributed to normalizing one aspect of HPA axis dysregulation observed in women with FM. The results are consistent with the findings from (1) an uncontrolled, multi-disciplinary CBT and aquatic exercise program for women with FM that showed a greater AUC for cortisol post-treatment, ${ }^{61}$ and (2) a non-randomized, but controlled, ten-week yoga program for women with rheumatoid arthritis that showed no change in the CAR post-treatment. ${ }^{38}$ The absence of a significant change in the CAR in the present study fits with the non-significant change in postintervention HADS depression scores in that an attenuated rise in the CAR has been related to greater subclinical 
depressive symptoms and a greater rise to milder symptoms. ${ }^{62}$ However, it is possible that a more sensitive measure of the $\mathrm{CAR}$ and/or a longer yoga intervention may be required to demonstrate improvements in the CAR and HADS depression scores.

In order to evaluate diurnal variability, we sampled cortisol three times daily precluding a more precise analysis, ${ }^{63}$ which requires a more intense sampling protocol of 5-7 samples over the course of the day, for two consecutive days. It is possible that with a greater number of measurements, differences in diurnal variability may have been observed. It is also possible that the absence of a significant difference from pre- to postintervention AUC, when using the protocol-compliant analysis, was due to a lack of power as significance was observed with the addition of the three participant's missing data. A methodology employing a greater number of sampling time points would also allow for the division of participants into HPA subgroups of typical, flat or inconsistent. ${ }^{42}$ Since FM is associated with variable HPA functioning as well as a high variability of illness profiles, ${ }^{3}$ such grouping would be very useful in determining a more accurate picture of HPA patterns in this population. Further grouping according to symptom subgroups may be most effective when evaluating the effects of a pain management program, ${ }^{64}$ and future research might consider the use of a larger sample size to allow for classification of FM participants according to sensory symptoms and comorbidities $^{65}$ within the context of a yoga intervention. Future research should measure other variables that are implicated in HPA function, such as growth hormone or inflammatory cytokines, to better understand how a yoga intervention might impact HPA function more broadly. Taken together, it is clear that randomized, controlled trials are needed to further examine the effect of mind-body practices, such as yoga, on cortisol in women with FM.

Speculation on the underlying mechanisms of yoga for chronic pain conditions includes physiological changes that impact the pain experience. ${ }^{25}$ Yoga promotes the relaxation response, which consists of a decreased heart rate, increased breath volume, and increased digestive function, amongst improvements on other physiological responses to stress, by increasing parasympathetic nervous system activity and decreasing sympathetic nervous system activity. ${ }^{66}$ Recent research indicates that advanced yoga practitioners have lower interleukin-6 levels than novice practitioners and it has been suggested that a regular yoga practice may serve to inhibit a pro-inflammatory response to stress. ${ }^{67}$ It is possible that such physiological changes may also have contributed to the post-yoga intervention changes we observed. Future research should examine the effects of yoga on these psycho-immune-endocrine interactions in FM.

The present yoga intervention did not result in improvements in the HADS depression and anxiety scores over the course of the yoga program. These findings are not consistent with other yoga studies of women with $\mathrm{FM}^{26}$ and rheumatoid arthritis. ${ }^{38}$ Reasons for this discrepancy include differences among the studies in the measures used to assess depressive symptomology (ie, Beck Depression Inventory, ${ }^{38}$ Revised Fibromyalgia Impact Questionnaire, ${ }^{26}$ HADS in the present study), the duration and intensity of the yoga program (10 weeks, ${ }^{38} 8$ weeks plus group discussion, ${ }^{26} 8$ weeks), or both. A longer intervention, including a social support component, may be needed to produce post-intervention improvements in depression as measured by the HADS.

There are limitations to the present study. The main methodological shortcoming is the absence of a control group, raising the possibility that the observed effects are not due to the yoga program per se but reflect spontaneous recovery, regression to the mean, and/or non-specific factors such as a caring atmosphere and attention paid to participants. In addition, the relatively small sample size limits statistical power and the absence of males limits the generalizability of the findings. Finally, notwithstanding the observed postintervention changes in total cortisol and diurnal variability, the absence of a change in the CAR may be a limitation of our salivary cortisol sampling procedure. More frequent sampling of salivary cortisol would also permit a more finegrained classification of HPA axis functioning.

In conclusion, the results of the present pilot study suggest that an eight-week yoga program helps to improve various psychological and pain related variables; namely, to decrease continuous pain and pain catastrophizing, to increase chronic pain acceptance and levels of mindfulness, as well as to alter cortisol levels in women with FM. Use of a randomized, controlled design with a larger sample size is recommended to better understand the effects of a yoga intervention on the above variables. A longer intervention may yield more robust findings, and a six-month follow-up may provide valuable information concerning the potential for lasting improvement.

\section{Acknowledgments}

We would like to thank Robin Hurlow and Claire Wicks for their expertise and commitment in the execution of the yoga program, and Dr Warren Nielson and Neil Pearson for their suggestions concerning fibromyalgia and appropriate asana practices for chronic pain, respectively. We would 
also like to thank Dr Rob Cribbie for his guidance in the statistical analysis. JK is supported by a Canadian Institutes of Health Research (CIHR) Canada Research Chair (CRC) in Health Psychology. KC was supported by a CIHR Frederick Banting and Charles Best Canada Graduate Scholarship - Master's Award and a CIHR Strategic Training Grant Fellowship in Pain: Molecules to Community. Funds to conduct this study were provided by JK's CIHR CRC in Health Psychology. This paper is derived, in part, from KC's Master's thesis.

\section{Disclosure}

The authors report no conflicts of interest in this work.

\section{References}

1. Goldenberg DL. Diagnosis and differential diagnosis of fibromyalgia. Am J Med. 2009;122(12 Suppl):S14-S21.

2. Wolfe F, Clauw DJ, Fitzcharles MA, et al. The American College of Rheumatology preliminary diagnostic criteria for fibromyalgia and measurement of symptom severity. Arthritis Care Res (Hoboken). 2010; 62(5):600-610.

3. Arnold LM. The pathophysiology, diagnosis and treatment of fibromyalgia. Psychiatr Clin North Am. 2010;33(2):375-408.

4. van Koulil S, van Lankveld W, Kraaimaat FW, van Riel PL, Evers AW. Risk factors for longer term psychological distress in well-functioning fibromyalgia patients: a prospective study into prognostic factors. Patient Educ Couns. 2009;80(1):126-129.

5. Fietta P, Fietta P, Manganelli P. Fibromyalgia and psychiatric disorders. Acta Biomed. 2007;78(2):88-95.

6. McDermid AJ, Rollman GB, McCain GA. Generalized hypervigilance in fibromyalgia: evidence of perceptual amplification. Pain. 1996; 66(2-3):133-144.

7. Nelson PJ, Tucker S. Developing an intervention to alter catastrophizing in persons with fibromyalgia. Orthop Nurs. 2006;25(3):205-214.

8. Martin MY, Bradley LA, Alexander RW, et al. Coping strategies predict disability in patients with primary fibromyalgia. Pain. 1996;68(1): 45-53.

9. Nicassio PM, Schoenfeld-Smith K, Radojevic V, Schuman C. Pain coping mechanisms in fibromyalgia: relationship to pain and functional outcomes. J Rheumatol. 1995;22(8):1552-1558.

10. Pruessner JC, Wolf OT, Hellhammer DH, et al. Free cortisol levels after awakening: a reliable biological marker for the assessment of adrenocortical activity. Life Sci. 1997;61(26):2539-2549.

11. Wingenfeld K, Heim C, Schmidt I, Wagner D, Meinlschmidt G, Hellhammer DH. HPA axis reactivity and lymphocyte glucocorticoid sensitivity in fibromyalgia syndrome and chronic pelvic pain. Psychosom Med. 2008;70(1):65-72.

12. Wingenfeld K, Nutzinger D, Kauth J, Hellhammer DH, Lautenbacher S. Salivary cortisol release and hypothalamic pituitary adrenal axis feedback sensitivity in fibromyalgia is associated with depression but not with pain. J Pain. 2010;11(11):1195-1202.

13. Riva R, Mork PJ, Westgaard RH, Ro M, Lundberg U. Fibromyalgia syndrome is associated with hypocortisolism. Int J Behav Med. 2010; 17(3):223-233.

14. Fries E, Hesse J, Hellhammer J, Hellhammer DH. A new view on hypocortisolism. Psychoneuroendocrinology. 2005;30(10): 1010-1016.

15. Izquierdo-Alvarez S, Bocos-Terraz JP, Bancalero-Flores JL, Pavon-Romero L, Serrano-Ostariz E, de Miquel CA. Is there an association between fibromyalgia and below-normal levels of urinary cortisol? BMC Res Notes. 2008;1:134.
16. Gur A, Cevik R, Sarac AJ, Colpan L, Em S. Hypothalamicpituitary-gonadal axis and cortisol in young women with primary fibromyalgia: the potential roles of depression, fatigue, and sleep disturbance in the occurrence of hypocortisolism. Ann Rheum Dis. 2004; 63(11):1504-1506.

17. McCain GA, Tilbe KS. Diurnal hormone variation in fibromyalgia syndrome: a comparison with rheumatoid arthritis. J Rheumatol Suppl. 1989;19:154-157.

18. Fabian LA, McGuire L, Page GG, Goodin BR, Edwards RR, Haythornthwaite J. The association of the cortisol awakening response with experimental pain ratings. Psychoneuroendocrinology. 2009;34(8): 1247-1251.

19. Kumari M, Badrick E, Chandola T, et al. Cortisol secretion and fatigue: associations in a community based cohort. Psychoneuroendocrinology. 2009;34(10):1476-1485.

20. O'Connor DB, Hendrickx H, Dadd T, et al. Cortisol awakening rise in middle-aged women in relation to psychological stress. Psychoneuroendocrinology. 2009;34(10):1486-1494.

21. Jones KD, Clark SR. Individualizing the exercise prescription for persons with fibromyalgia. Rheum Dis Clin North Am. 2002;28(2):419436, $x-x i$.

22. Kadetoff D, Kosek E. Evidence of reduced sympatho-adrenal and hypothalamic-pituitary activity during static muscular work in patients with fibromyalgia. J Rehabil Med. 2010;42(8):765-772.

23. Valkeinen H, Hakkinen K, Pakarinen A, et al. Muscle hypertrophy, strength development, and serum hormones during strength training in elderly women with fibromyalgia. Scand J Rheumatol. 2005;34(4): 309-314.

24. Giske L, Vollestad NK, Mengshoel AM, Jensen J, Knardahl S, Roe C. Attenuated adrenergic responses to exercise in women with fibromyalgia - a controlled study. Eur J Pain. 2008;12(3):351-360.

25. Wren AA, Wright MA, Carson JW, Keefe FJ. Yoga for persistent pain: new findings and directions for an ancient practice. Pain. 2011;152(3): $477-480$.

26. Carson JW, Carson KM, Jones KD, Bennett RM, Wright CL, Mist SD. A pilot randomized controlled trial of the Yoga of Awareness program in the management of fibromyalgia. Pain. 2010;151(2):530-539.

27. da Silva GD, Lorenzi-Filho G, Lage, LV. Effects of yoga and the addition of tui na in patients with fibromyalgia. J Alt Complement Med. 2007;13(10):1107-1113.

28. Kabat-Zinn J. Wherever You Go, There You Are: Mindfulness Meditation For Everyday Life. New York: Hyperion; 1994.

29. Sephton SE, Salmon P, Weissbecker I, et al. Mindfulness meditation alleviates depressive symptoms in women with fibromyalgia: results of a randomized clinical trial. Arthritis Rheum. 15 2007;57(1):77-85.

30. Lush E, Salmon P, Floyd A, Studts JL, Weissbecker I, Sephton SE. Mindfulness meditation for symptom reduction in fibromyalgia: psychophysiological correlates. J Clin Psychol Med Settings. 2009;16(2): 200-207.

31. Zautra AJ, Fasman R, Davis MC, Craig AD. The effects of slow breathing on affective responses to pain stimuli: an experimental study. Pain. 2010;149(1):12-18.

32. Vera FM, Manzaneque JM, Maldonado EF, et al. Subjective sleep quality and hormonal modulation in long-term yoga practitioners. Biol Psychol. 2009;81(3):164-168.

33. Woolery A, Myers H, Sternlieb B, Zeltzer L. A yoga intervention for young adults with elevated symptoms of depression. Altern Ther Health Med. 2004;10(2):60-63.

34. West J, Otte C, Geher K, Johnson J, Mohr DC. Effects of Hatha yoga and African dance on perceived stress, affect, and salivary cortisol. Ann Behav Med. 2004;28(2):114-118.

35. Kamei T, Toriumi Y, Kimura H, Ohno S, Kumano H, Kimura K. Decrease in serum cortisol during yoga exercise is correlated with alpha wave activation. Percept Mot Skills. 2000;90(3 Pt 1):1027-1032.

36. Michalsen A, Grossman P, Acil A, et al. Rapid stress reduction and anxiolysis among distressed women as a consequence of a three-month intensive yoga program. Med Sci Monit. 2005;11(12):CR555-CR561. 
37. Vedamurthachar A, Janakiramaiah N, Hegde JM, et al. Antidepressant efficacy and hormonal effects of Sudarshana Kriya Yoga (SKY) in alcohol dependent individuals. J Affect Disord. 2006;94(1-3): 249-253.

38. Bosch PR, Traustadottir T, Howard P, Matt KS. Functional and physiological effects of yoga in women with rheumatoid arthritis: a pilot study. Altern Ther Health Med. 2009;15(4):24-31.

39. Carlson LE, Speca M, Patel KD, Goodey E. Mindfulness-based stress reduction in relation to quality of life, mood, symptoms of stress and levels of cortisol, dehydroepiandrosterone sulfate (DHEAS) and melatonin in breast and prostate cancer outpatients. Psychoneuroendocrinology. 2004;29(4):448-474.

40. Granath J, Ingvarsson S, von Thiele U, Lundberg U. Stress management: a randomized study of cognitive behavioural therapy and yoga. Cogn Behav Ther. 2006;35(1):3-10.

41. Centre for Studies on Human Stress. How To Measure Stress in Humans. Quebec, Canada: Fernand-Sequin Research Centre of Louis H. Lafontaine Hospital; 2007.

42. Smyth JM, Ockenfels MC, Gorin AA, et al. Individual differences in the diurnal cycle of cortisol. Psychoneuroendocrinology. 1997;22(2): 89-105.

43. Raub JA. Psychophysiologic effects of Hatha Yoga on musculoskeletal and cardiopulmonary function: a literature review. JAltern Complement Med. 2002;8(6):797-812.

44. Pearson N. Yoga for people in pain. International Journal of Yoga Therapy. 2008;18:77-86.

45. Dworkin RH, Turk DC, Revicki DA, et al. Development and initial validation of an expanded and revised version of the Short-form McGill Pain Questionnaire (SF-MPQ-2). Pain. 2009;144(1-2):35-42.

46. Jensen MP, Karoly P. Self-report scales and procedures for assessing pain in adults. In Turk CD, Melzack R, editors. Handbook of Pain Assessment. Third ed. New York: The Guilford Press; 2011.

47. Staud R, Price DD, Robinson ME, Vierck CJ Jr. Body pain area and pain-related negative affect predict clinical pain intensity in patients with fibromyalgia. J Pain. 2004;5(6):338-343.

48. Sullivan MJ, Bishop SC, Pivik J. The Pain Catastrophizing scale: development and validation. Psychological Assessment. 1995;7: 524-532.

49. Tait RC, Chibnall JT, Krause S. The Pain Disability Index: psychometric properties. Pain. 1990;40(2):171-182.

50. Zigmund AS, Snaith RP. The hospital anxiety and depression scale. Acta Psychiatr Scand. 1983;67(6):361-370.

51. Bjelland I, Dahl AA, Haug TT, Neckelmann D. The validity of the Hospital Anxiety and Depression Scale. An updated literature review. J Psychosom Res. 2002;52(2):69-77.

52. Baer RA, Smith GT, Hopkins J, Krietemeyer J, Toney L. Using selfreport assessment methods to explore facets of mindfulness. Assessment. 2006;13(1):27-45.
53. Baer RA, Smith GT, Lykins E, et al. Construct validity of the five facet mindfulness questionnaire in meditating and nonmeditating samples. Assessment. 2008;15(3):329-342.

54. McCracken LM, Vowles KE, Eccleston C. Acceptance of chronic pain: component analysis and a revised assessment method. Pain. 2004;107(1-2):159-166.

55. Pruessner JC, Kirschbaum C, Meinlschmid G, Hellhammer DH. Two formulas for computation of the area under the curve represent measures of total hormone concentration versus time-dependent change. Psychoneuroendocrinology. 2003;28(7):916-931.

56. Roberts AD, Charler ML, Papadopoulos A, Wessely S, Chalder T, Cleare AJ. Does hypocortisolism predict a poor response to cognitive behavioural therapy in chronic fatigue syndrome? Psychol Med. 2010;40(3):515-522.

57. Fields A. Discovering Statistics Using SPSS. Third ed. London: Sage; 2009.

58. Preacher KJ, Hayes AF. SPSS and SAS procedures for estimating indirect effects in simple mediation models. Behav Res Methods Instrum Comput. 2004;36(4):717-731.

59. Shapiro SL, Carlson LE, Astin JA, Freedman B. Mechanisms of mindfulness. J Clin Psychol. 2006;62(3):373-386.

60. Turk DC, Wilson HD. Fear of pain as a prognostic factor in chronic pain: conceptual models, assessment, and treatment implications. Curr Pain Headache Rep. 2010;14(2):88-95.

61. Bonifazi M, Suman AL, Cambiaggi C, et al. Changes in salivary cortisol and corticosteroid receptor-alpha mRNA expression following a 3-week multidisciplinary treatment program in patients with fibromyalgia. Psychoneuroendocrinology. 2006;31(9):1076-1086.

62. Mangold D, Marino E, Javors M. The cortisol awakening response predicts subclinical depressive symptomatology in Mexican American adults. J Psychiatr Res. 2011;45(7):902-909.

63. Adam EK, Kumari M. Assessing salivary cortisol in large-scale, epidemiological research. Psychoneuroendocrinology. 2009;34(10): 1423-1436.

64. Verra ML, Angst F, Brioschi R, et al. Does classification of persons with fibromyalgia into Multidimensional Pain Inventory subgroups detect differences in outcome after a standard chronic pain management program? Pain Res Manag. 2009;14(6):445-453.

65. Rehm SE, Koroschetz J, Gockel U, et al. A cross-sectional survey of 3035 patients with fibromyalgia: subgroups of patients with typical comorbidities and sensory symptom profiles. Rheumatology (Oxford). 2010;49(6):1146-1152.

66. Dunn KD. A review of the literature examining the physiological processes underlying the therapeutic benefits of Hatha yoga. Adv Mind Body Med. 2008;23(3):10-18.

67. Kiecolt-Glaser JK, Christian L, Preston H, et al. Stress, inflammation, and yoga practice. Psychosom Med. 2010;72(2):113-121.
Journal of Pain Research

\section{Publish your work in this journal}

The Journal of Pain Research is an international, peer-reviewed, open access, online journal that welcomes laboratory and clinical findings in the fields of pain research and the prevention and management of pain. Original research, reviews, symposium reports, hypothesis formation and commentaries are all considered for publication.

\section{Dovepress}

The manuscript management system is completely online and includes a very quick and fair peer-review system, which is all easy to use. Visit http://www.dovepress.com/testimonials.php to read real quotes from published authors. 